\title{
Das Beginenwesen der sächsisch-thüringischen Lande in seiner sozialen Bedeutung.
}

\author{
Von GEORG LIEBE.
}

Je mehr wir gewohnt sind, die soziale Fürsorge im Mittelalter einzig von der Kirche gepflegt, alle ihre Massnahmen auf religiöse Beweggründe zurückgeführt zu sehen, desto bemerkenswerter erscheinen uns Einrichtungen, in denen einzig das Bestreben, einem Notstande abzuhelfen, zweckmässige Gestaltung fand. Auch bei dem Beginenwesen pflegt dem religiösen Element ein unverhāltnismässiges Gewicht beigemessen zu werden: in der Tat hat es keine grössere Rolle dabei gespielt ais bei allen genossenschaftlichen Bildungen der Zeit wie den Bruderschaften der Handwerker. Ist es doch ein reines Erzeugnis der städtischen Kultur, die von der Wurzel an antikirchlich gewesen ist. Fast immer werden die Beginenhăuser nach den Stiftern genannt, denen sie ihre Existenz verdanken, nicht nach Heiligen, und die Stadtmagistrate traten einem Übergreifen der geistlichen Aufsicht wie bei den Hospitälern stets entgegen, wie denn die Beginen auch bedepflichtig waren.

Der Ausgangspunkt der weit verbreiteten Einrichtung war ein Notstand, der nur auf dem Boden der Kultur, besonders der städtischen, zu wuchern pflegt: die Unmöglichkeit, die weiblichen Mitglieder der Gesellschaft in der Ehe zu versorgen. War doch im Mittelalter das Missverhältnis der Geschlechter weit schreiender als jetzt wegen der zahlreichen Bedrohungen, denen die öfentliche Unsicherheit das Leben der Männer aussetzte, und des Cölibats der zahlreichen Geistlichkeit. Es ist anzunehmen, dass die starke Verminderung des männlichen Elements durch die Kreuzzüge den ersten Anstoss gegeben hat. Die erste Organisation 
wird dem Priester Lambert le Bègue (Stammler) zugeschrieben, der $1184 \mathrm{zu}$ Lüttich eine Stiftung zur Versorgung alleinstehender Frauen ins Leben rief. Durch diese Annahme würde sich auch am ungezwungensten die Entstehung des in den mannigfachsten Formen auftretenden Namens erklären, wobei ein schon früheres Bestehen derartiger Institute nicht ausgeschlossen ist. Auch spāter werden nicht selten noch allgemeine Bezeichnungen wie Schwestern oder Kinder verwendet. Die besonders reiche Entfaltung und zähe Erhaltung des Beginenwesens in den Niederlanden spricht jedenfalls für ein Entstehen in dieser alten Kulturlandschaft. Auch in Deutschland, wo es im dreizehnten und vierzehnten Jahrhundert seine reichste Blūte sah, knūpft es sich an den Gang der stādtischen Kultur, treibt es die zahlreichsten Sprossen in den grossen Handelssitzen des Westens, von denen Köln 106, Frankfurt 54, Strassburg 60, Basel 30 Beginenhāuser in seinen Mauern sah, und nimmt nach Osten und Norden mit den Stādten an Bedeutung ab. Unter einer zu gesteigerter Tătigkeit und Genusssucht gedrāngten Bevölkerung musste die Unsicherheit des weiblichen Geschlechts, die das alte Strafrecht so grell beleuchtet, am dringendsten zu Schutzmassregeln mahnen.

Bei aller weiten Verbreitung lässt die Organisation des Beginenwesens eine grosse Gleichmāssigkeit erkennen, die von den sich überall gleich bleibenden Gründen seiner Entstehung zeugt. Stets sind es Vereinigungen von Frauenspersonen, die sich in einer Zahl von meist zwei bis fünfzehn zusammengetan haben, um im Zusammenleben Schutz und leichtere Existenzbedingungen zu finden. Die Grundlage dieser als Konvente, Einungen, Gotteshăuser, auch blos Hāuser bezeichneten Vereinigungen war regelmässig die Stiftung eines Wohltäters, doch waren besonders in der ältern Zeit wohlhabende Mitglieder nicht selten, deren Vermögen der Gesamtheit zu gute kam. Machte die nach aussen abgeschlossene Gemeinsamkeit des Wohnens unter selbstgewählten Vorsteherinnen die Beginen allerdings den Nonnen ähnlich, so hatten sie doch ausser ehrbarem Wandel keine andern religiösen Pflichten als jede fromme Frau. Zur Vermögensverwaltung pflegte der Rat ihnen wie den Hospitälern Beistānde aus seiner Mitte zu verordnen. 
Neben den Rheinlanden ist das Beginenwesen in den alten städtischen Niederlassungen Sachsen-Thüringens wenn auch nicht zu gleicher Verbreitung, so doch zu reicher Ausbildung gelangt. Jedenfalls weist es schon auf die gleiche Einrichtung hin, wenn 1238 drei Schwestern nebst der Tochter der einen und einer Magd in Erfurt als convivae und deo dicatae bezeichnet werden gelegentlich der Schenkung einer Hufe an das Predigerkloster unter Vorbehalt der Nutzniessung. Gleicherweise erscheinen $1257 \mathrm{zw}$ ei sorores carnales et habitu spiritales mit der Schenkung einer halben Hufe an das Marienstift, erst 1272 kommt die Benennung Begine vor - diese Namensform ist die in der Landschaft gebräuchliche. Doch wird noch 1274, 1283, 1298 die Bezeichnung soror, swester gebraucht, ebenso 1281 zu Hildesheim. ${ }^{1)}$ Erst um 1300 wird der Name Begine zur Regel. Die rasch wachsende Zahl der Neugrūndungen zeigt, wie sehr der Gedanke dem Bedūrfnis der Zeit entsprach. Eine poetische Schilderung Erfurts um 1282 nennt die dortigen Beginen sehr zahlreich, 1308 erscheint eine Begine Richardis als Begründerin eines Konvents im S. Pauls-Kirchspiel und 1353 macht Tile von der Sachsa dem Konvent am Nunnensack (nahe dem Predigerkloster) ein Vermāchtnis. Der 1281 in Hildesheim genannte Konvent (im hintern Brühl) wird 1352, 1356 der neue genannt, sodass er also schon bei jener ersten Erwāhnung einen Vorgānger gehabt haben muss, in Halberstadt lässt 1302 die Bezeichnung maior conventus auf das Bestehen mehrerer schliessen, und in Braunschweig sind dem ersten 1290 erwāhnten bis 1350 fünf weitere gefolgt. ?

Ihrer Herkunft nach müssen die Insassinnen dieser ersten Hāuser vielfach den höheren Stānden angehört haben, erscheinen sie ja gerade in den ersten Urkunden als Schenkerinnen an geist-

1) Urkundenbuch der Stadt Erfurt ed. Beyer I nr. 117, 164, 268, 466; Urkundenbuch des Klosters Pforte ed. Böhme nr. 238; Urkundenbuch der Stadt Hildesheim ed. Doebner 1 or. 374; auch in Bremen wird 1266, 1268, 1300 sorores, 1278 bachinae gebraucht (Urkundenbuch ed. Ehmck u. v. Bippen I nr. 325, 337, 382, 538).

2) Nicolai de Bibre carmen satiricum ed. Fischer v. 1605; UB. v. Hildesheim II nr. 76, 118; UB. v. Erfurt I nr. 540, II nr. 404; UB. d. Stadt Halberstadt ed. Schmidt nr. 294 ; Rehtmeyer, Kirchengeschichte d. Stadt Braunschweig S. 215; in Wismar ist 1287 von zwei Hãusern die Rede (Meklenburgisches Urkundenbuch III nr. 1908), in Frankfurt a. M., wo die ersten Beckinen 1280 genannt werden, gab es um die Mitte des 14. Jahrhunderts mindestens 16 Konvente. (Kriegk, Deutsches Bürgertum I S. 107, 109.) 
liche Anstalten, und ähnliche Beobachtungen lassen sich auch fernerhin machen. In Erfurt erscheinen aus der Gefrundenfamilie von Schwerstedt 1301 Margarete, 1308 Irmentrud und Gertrud als Beginen, erstere verpfāndet die Hälfte ihres ererbten Hofes für 25 Pfund dem Marienstift, letztere kaufen für 27 Pfund eine Hufe, 1305 verschreibt der Pfarrer der Michaeliskirche seiner Mutter und deren Schwester, einer Begine, die Nutzniessung einer halben Hufe, 1332 bekennt sich Markgraf Günther zu Kindelbrück als Schuldner einer Begine Jutta von Nordhausen für einen Hufenzins von sechs Malter Korn, 1350 konsentiert die Begine Jutta, Tochter eines Ritters Heinrich, zu einem Verkauf ihrer Mutter. ${ }^{3}$ ) In Halberstadt ūbertrāgt 1306 Schwester Zacharia der Martinikirche eine Bude gegen bestimmte Leistungen an ihren Konvent, 1310 ermahnt der Offizial das Kloster Hecklingen, seiner Zinsverpflichtung gegen die Begine Mechthild nachzukommen. ") In Mūhlhausen behălt 1309 die Begine Christine von Schönstedt die Nutzniessung des von ihrem Vater dem Predigerorden geschenkten Vermögens, ebenso 1311 zwei andre von Grundstücken, die nach ihrem Tode dem Antoniusspital zufallen sollen, und 1318 verkaufen zwei Beginen aus der Patrizierfamilie von Ammern eine halbe Hufe dem Deutschordenshause. ${ }^{5}$ ) Vorzugsweise diese aus günstigen Verhältnissen stammenden Mitglieder werden von der Erlaubnis des Austritts Gebrauch gemacht haben, die das Beginenwesen von allen klösterlichen Einrichtungen grundsātzlich schied. 1318 gestattet Erzbischof Peter von Mainz einer Erfurter Matrone, das von ihr als Begine bewohnte, jetzt ihın gehörige Haus weiter zu bewohnen, 1319 schenkt eine frühere Begine der dortigen Bartholomäuskirche eine Hufe mit Vorbehalt einer Rente, 1328 schenken zwei frühere Beginen aus der Mühlhäuser Patrizierfamilie von Seebach demı Deutschordenshaus eine halbe Hufe, 1337 zwei solche aus der Patrizierfamilie von Bolkstedt dem Kloster Volkerode einen Hof, 1349 vertauschen drei leibliche Schwestern eine Hufe. ') Auch die

3) UB. v. Erfurt I nr. 495, 549, 521, II nr. 107, 343.

4) UB. v. Halberstadt nr. 313, Heinemann, Codex dipl. Anhalt, III nr. 221.

) Urkundenbuch der Stadt Mühlhausen ed. Herquet nr. 608, 624, 726; 1317 er-

werben in Worms zwei Beginen eine Hypothek (UB. d. Stadt Worms ed. Boos $11 \mathrm{nr}$. 128).

G) UB. von Erfurt I nr. 620, 624; UB, von Mühlhausen nr. 827, 897, 1016. 
nicht seltenen Bestimmungen der Statuten über den völligen oder teilweisen Vermögensverzicht einer austretenden Begine zeigen, wie man mit dem Vorhandensein von Vermögen rechnete.

Haben unter solchen Umstānden besonders in der āltern Zeit einzelne Hăuser einen exklusiven Charakter angenommen, wovon sich in Strassburg, Frankfurt, Wesel Spuren finden, so liegt es doch in der Natur der Sache, dass bei den Stiftungen mehr und mehr mit der Armut der Aufzunehmenden gerechnet wurde. Es war dies um so wesentlicher, je mehr die wachsenden Ansprüche der Klöster diese nur den Töchtern der Wohlhabenden zugänglich machten; dafür boten aber die Beginenhäuser nicht die Aussicht auf ein sorgenloses Dasein, sondern legten die Verpflichtung zur Arbeit auf. Die oben genannte Erfurter Urkunde von 1257, wahrscheinlich von zwei Beginen ausgestelit, bezeichnet die von ihnen geschenkte halbe Hufe als durch ihrer Hānde Arbeit erworben. Allgemein befolgte man den Grundsatz, den die Statuten eines Konvents zu Wesel 1309 aussprechen: Die Aufzunehmende solle Vermögen besitzen oder eine Kunst verstehen. ') Als solche bot sich am natürlichsten die von Alters her eine Domāne der Frauen bildende Textilindustrie. Wie wir überall im zünftigen Weberhandwerk Frauen tătig finden, meist als Lohnarbeiterinnen, nicht selten aber auch selbständig, so tritt das Spinnen und Weben als ständige Beschäftigung der Beginen auf. Konnte ihnen dieselbe zum eignen Bedarf nicht verwehrt werden, so führte dagegen der Handel, den sie mit dem darüber hinaus Produzierten trieben, frühzeitig zu Streitigkeiten mit der Zunftgerechtsame, ähnlich wie der mit eigenem Gewāchs betriebene Weinschank der Geistlichkeit fortdauernd einen Zankapfel bildete. Mit Rücksicht auf den von den Beginen betriebenen Handel mit Wollenwaren gestattete 1282 Erzbischof Werner von Mainz dem Erfurter Rat, sie zu den bürgerlichen Lasten heranzuziehen, und die erwähnte gleichzeitige Schilderung des Nikolaus von Bibra weiss gleichfalls davon zu sagen: Jeiunant, vigilant et lanea stamina filant. Dieser Handel muss eine ziemliche Ausdehnung gewonnen haben, denn 1332 wird von den Tuchabfällen der Gewandschneider

i) Heidemann, Die Beguinenhāuser Wesels (Zschr. d. Bergischen Geschichtsvereins 1867 S. 94). 
und Beginen, die wieder zur Fabrikation verwendet werden, ein Zoll erhoben, den das s. g. Bibrabüchlein unter die mainzischen Gefälle rechnet. Den Würzburger Beginen wurde 1293 der Handel mit selbstgewebten Stoffen vom Bischof freigegeben und in demselben Jahre bei dem Beginenhaus in Wismar ein Bleichplatz erwāhnt, wie er bei den niederrheinischen Beginenhäusern die Rege! bildet. Dagegen untersagte 1321 der Rat von Zerbst den Beginen Tuchweberei und -ausschnitt. ?)

Unbestrittener als diese gewerbliche Tätigkeit blieb das Gebiet der Krankenpflege. Sie übten diese teils den Diakonissinnen vergleichbar in den Hãusern aus, wie es aus Frankfurt und Worms überliefert ist, teils bildeten sie das ständige Pflegepersonal der Spitäler. So werden in Goslar 1274 und 1295 Beginen als wohnhaft im Ludwigsspital erwāhnt, und das gleiche ist anzunehmen, wenn 1309 ein Freiberger Bürger eine Jahresrente an das Spital und eine Begine schenkt. Das 1351 zu Braunschweig gegründete Spital S. Jodoci hatte Beginen als Pflegerinnen, und in Hildesheim wird später als eine ihrer Niederlassungen das Johannisspital genannt. Daneben wird auch, z. B. in Wesel, erwähnt, dass sie sich armer, besonders verwaister Mādchen angenommen hătten. ${ }^{9}$ )

$\mathrm{Da}$ den Beginen nur sittliche, keine besondere Ordenspflichten oblagen, waren ihre Statuten, besonders in früherer Zeit, sehr einfach. $\mathrm{Zu}$ den ältesten erhaltenen gehören die des von dem Wormser Bürger Gudelmann gestifteten Hauses von 1288, die Braunschweiger von 1290, die Halberstādter von 1302 und 1316, die Hildesheimer von 1326, die Weseler von 1309 und 1326. ${ }^{10}$; Teils von den Stiftern unter Konsens der Stadtbehörden, teils von den geistlichen Autoritāten, vornehmlich den Bischöfen erlassen, pflegen sich diese ältern Ordnungen kurz über die Eigentumsverhältnisse und das Zusammenleben der Schwestern zu verbreiten. Strenge Gemeinsamkeit des Besitzes, die sich meist auch

8) UB. von Erfurt I nr. 325; Carmen satiricum v. 1619; Kirchhoff, Die ältesten Weistūmer der Stadt Erfurt, 1870 S. 113; Rost, Beguinen im Fürstentum Würzburg, 1846 S. 22; Meklenburg. UB. III nr. 2217; Peter Beckers Chronik von Zerbst ed. Kindscher S. 130

9) Urkusderbuch d. Stadt Goslar ed. Bode II nr. 203, 484; Urkundenbuch d. Stadt Freiberg ed. Ermisch (Cod. dipl, Saxon.) I nr. 59; Dürre, Geschichte der Stadt Braunsch veig S. 596, Heidemann a. a. O. S. 87.

10) UB. v. Worms I, Dürre a. a. O. S. 598, UB. von Halberstadt nr. 294, 359: UB. von Hildesheim I nr. 374, 771 ; Heidemann a. 2. O. S. 93, 91. 
auf das Vermögen der Austretenden erstreckt, wird eingeschärft, und die stets wiederkehrende Ermahnung zur Friedfertigkeit nebst Androhung der Ausstossung zeigt, wie deutlich man die Gefahren Jes gemeinsamen Haushalts erkannte. Auch auf die Abwendung sittlicher Gefāhrdung war man frūh bedacht, wie es die erwāhnte Erfurter Schilderung ausspricht:

Dicite begine: soror esto domi, quia Dine

Si clam sedisset, non vis illata fuisset

Sed nec adhuc fleret, quod virginitate careret. ${ }^{11}$ )

Die Ausgänge der Schwestern und das Betreten des Hauses durch Männer unterlagen daher strenger Kontrolle. Zur Handhabung der Disziplin war eine Vorsteherin unter dem Titel Meisterin bestellt, welche die Hausgenossinnen in der Regel selbst wählten, wie sie sich auch durch Kooptation ergānzten. Trotz aller Vorsichtsmassregeln waren ärgerliche Vorgänge nicht $z u$ verhüten, und schon 1244 griff eine Synode zu Fritzlar zu dem drastischen Mittel, die Aufnahme von Schwestern unter vierzig Jahren zu verbieten, wovon der Erzbischof von Mainz 1291 die Frankfurter Hāuser ausdrücklich entband. Die Schilderung freilich, welche der Erfurter Kleriker Nikolaus von Bibra von den dortigen Zuständen entwirft, entstammt in ihrem pastosen Farbenauftrag wohl grossenteils seiner auch sonst bekannten Neigung zu behaglichem Cynismus. ${ }^{11}$ ) Nachdem er das eingezogene Leben der frommen Beginen gepriesen, geisselt er die andern, die sich umhertreiben, auf dem Markte, in den Klöstern und in Gesellschaft der Schūler zu finden sind:

Hic declinare discunt et metra parare

Sed neque spondeum curant nec habere trocheum

Tercius ancillis tantum pes congrait illis.

Hoc, bene si recolo, cudunt pede carmina solo.

Wenn er des weiteren auch vor der Ausmalung der Folgen - Aussetzung und Kindesmord - nicht zurūckschreckt, so stand er mit solchen Anschauungen keineswegs vereinzelt da, denn eine gleichzeitige Satire bemerkt mit drastischer Trockenheit:

11) Carmen satir. v. 1294, 1628, Vorrede S. 21, Cod. dipl. Moenofranoof. ed. Jjöhmer S. 262. 
Vix etiam quevis sterilis reperitur in illis

Donec eis etas talia posse negat.

Indessen waren es keineswegs diese wie es scheint doch nur ausnahmsweise hervortretenden sittlichen Mängel, welche im vierzehnten Jahrhundert die Obrigkeiten zum Vorgehen gegen die Beginenhäuser veranlassten, sondern vielmehr ihre selbstāndige Stellung gegenüber der Kirche. Von Anbeginn hatten ihre Angehörigen zwar auf strenge Erfüllung der kirchlichen Pflichten gehalten, der Geistlichkeit gegenüber aber durchaus ihre Unabhängigkeit gewahrt und dabei die Unterstūtzung der Kommunalbehörden gefunden. Wurzelten doch diese Anstalten recht eigentlich in städtischem Boden, von reichen Bürgern gestiftet und zu Zufluchtsstätten fūr Stadtkinder bestimmt. Es findet sich daher auch nirgends eine besondere Ordenstracht der Beginen, denen nur die auusserste Einfachheit geboten war, wie sie sich bei der häufigen Verwendung selbstgefertigter Stoffe von selbst verstand. Die Bezeichnung graue oder blaue Schwestern weist auf Bevorzugung dunkler Farben, daneben werden weisse Kopftūcher erwähnt. Zunächst fand sich die Kirche mit dem ab, was ihr eingerãumt wurde. Die Magdeburger Synodalstatuten von 1266 fordern von den Beginen nur Gehorsam gegen den Pfarrer ihrer Parochie gleich den übrigen Pfarrkindern - ein Beschluss, auf welchen noch 1295 Bischof Bernhard von Meissen die Pfarrer seiner Diōzese hinweist. Bischof Siegfried II von Hildeshein nimmt 1281 den dortigen Konvent Meienberg in seinen Schutz und sein Nachfolger Otto II. nennt dessen Insassen 1326 dilectae in Christo filiae. ${ }^{22}$;

Der innere Grund für die Verfolgungen, denen wir die Beginen kirchlicherseits im vierzehnten Jahrhundert wiederholt ausgesetzt sehen, war wohl die Verschärfung des Verhältnisses zwischen Klerus und Bürgerschaft, deren jeder seine Interessensphäre auf Kosten des anderen zu erweitern strebte. Mit zunehmender politischer Reife wollte die Stadtgemeinde nicht mehr eine Sonderexistenz in ihrer Mitte dulden, daher die Bemühungen, der toten Hand zu wehren, den Klerus zum Ungeld heranzuziehen,

27) Lünig, Reichsarchiv XX S. 259, Urkundenbuch des Hochstifts Meissen ed. Cier;dorf, Cod. dipl. Sax. I nr. 317, UB. v. Hildesheim 1 nr. 374, 771. 
über Kirchenvermögen und Schule sich die Kontrolle zu sichern. Gegen die Beginenhäuser, die vom Rat geschützten und in breiten Kreisen volkstümlichen, bot eine Handhabe der gefährliche Vorwurf der Ketzerei. Allerdings mussten in Zeiten, da alles geistige Leben nach der religiösen Seite gravitierte, diese abgeschlossenen Vereinigungen religiös gerichteter, aber durch keine Regel gebundener Frauen allen Gefahren des Konventikelwresens ausgesetzt sein. Die schwärmerische Mystik, welche damals die Gemüter beherrschte, musste bei der geringen Bildung der Mehrzahl einen unklaren Charakter annehmen und dem Einfluss von allerlei nicht immer unbedenklichen Sekten, wie der Brüder des freien Geistes, den Boden bereiten. Kaum aber hätten bei dem zurückgezogenen Leben der Beginen diese Beobachtungen zu einem so schroffen Vorgehen gegen sie geführt, wenn man sie nicht mit den Begarden zusammengeworfen hätte, die weit später auftauchend und ingleich geringer an Zahl, gleichgerichtete Vereinigungen von Männern darstellten. Keineswegs in dem Masse wie die Schwestern einem sozialen Bedürfnis entsprechend, sind sie weit häufiger auf Abwege geraten; wir hören Klagen, dass sie im Lande umherzogen und in heimlichen Versammlungen ihre Irrlehren vortrugen, sodass man kirchlicherseits dahin gelangte, sie als ketzerische Sekte gleich andern zu betrachten. Von dieser Auffassung geleitet verdammte Papst Klemens V. auf dem Konzil zu Vienne die Irrtümer der Begarden und Beginen. Danach lehrten sie, dass der Mensch schon in diesem Leben $\mathrm{zu}$ solcher Vollkommenheit gelangen könne, dass er nicht mehr zu sündigen vermöge und dem Körper gestatten dürfe, was ihm gefiele; der Kuss einer Frau sei Todsünde, der Geschlechtsakt aber nicht, weil zu jenem die Natur nicht neige, wohl aber zu diesem. ${ }^{13}$ ) Indessen war sich die Kurie wohl bewusst, dass solche Ausartungen einzelner der grossen Mehrzahl nicht zum Vorwurf gemacht werden konnten, und Papst Klemens V. wie Johann XXII., als er 1316 die Konzilsbeschlūsse von Vienne verkūndigte, nahmen ausdrücklich diejenigen Beginen aus, die ehrbar lebten und der Kirche Achtung erwiesen. Es kam überall darauf an, ob sich in den einzelnen Landschaften die

23) vgl. Wigger, Urkundliche Mitteilungen über die Beghinen- und Beghardenhäuser 2u Rostock. 1882 
Neigung und die Macht fand, von der gebotenen Waffe Gebrauch zu machen, und das wird naturgemäss da der Fall gewesen sein, wo eine grössere Anzahl der Angefeindeten die Opposition herausforderte. Dies war, wie wir gesehen haben, in Niedersachsen und Thüringen der Fall, und es sind daher auch Verfolgungen bezeugt. Zum Jahre 1319 berichtet die Magdeburger Schöffenchronik: „In dissem jare verbannede man die beginen unde baggarde, des nemen orer vele knechte und man, de vor kuschheit hatten gelovet. ${ }^{14}$ ) Man scheint sich also mit der Auflösung der Konvente begnügt und diese nicht sonderlich nachhaltig betrieben zu haben, da fünfzig Jahre spāter der Übereifer eines Ketzerrichters auf demselben Boden neue Nahrung fand. Es war der Erfurter Dominikaner Walter Kerlinger, der, vom Papste dazu bestellt, in seiner Heimat eine erbitterte Verfolgung gegen die vermeintlichen Ketzer ins Werk setzte. Im Jahre 1368 liessen sich nach der Erzählung in Detmars Chronik von den vierhundert in Erfurt lebenden zweihundert zum Austritt bewegen, die andern verfielen dem Bann und der Vertreibung, zwei Begarden wurden verbrannt. Mit gleichem Erfolge setzte der Inquisitor nebst drei Ordensbrūdern seine Tătigkeit in der magdeburgischen und bremischen Kirchenprovinz, den thüringischen, sächsischen und hessischen Landen fort. In Eisenach verlieh im selben Jahre der Landgraf Friedrich Balthasar und Wilhelm das Haus Tabernakel in der nach ihnen benannten Nonnengasse, "daraus der Konger - offenbar verderbt aus Kerlinger -., der Ketzermeister die Beginen hat vertrieben, “ einem Pfeifer. Das Jahr darauf soll nach Korners Bericht der fanatische Mönch zu Nordhausen sieben Ketzer haben verbrennen lassen und über dreissig Bussen auferlegt haben. Kaiser Karl IV. war mit seiner Wirksamkeit so zufrieden, dass er ihn in einer am 10. Juni 1369 zu Lucca ausgestellten Urkunde ermăchtigte, die Hăuser der Begarden zu Untersuchungsgefängnissen zu verwenden, die der Beginen zu verāussern, teils den Inquisitoren, teils den Armen zum Besten. ${ }^{15}$ ) Ganz anders als die höchste kirchliche und staatliche Autorităt war aber offenbar die Volksmeinung gesonnen. Sie klingt aus der Erfurtei

14) Magdeburger Schöffenchronik ed. Janicke, Seite 174.

15) Thüringische Zeitschrift IV, S. 227, Wigger a. 2. O. Anhang. 
S. Peterschronik wieder, die Pabst Clemens auf seinem Sterbebette 1314 sein Vorgehen bereuen lässt, ${ }^{10}$ ) und Detmar bemerkt anlāsslich der Verfolgung von 1368 gradezu: "De lude helden mer von en dan van aller geistliken achte; des wart men wol war, do men se vorhorde."

An ihrer Volkstümlichkeit fanden die Beginenkonvente den Rückhalt, der sie die Stürme der Verfolgung überdauern liess. Der sicherste Beweis dafür ist, dass fortwährend neue Häuser gestiftet wurden; aber innerhalb derselben scheint sich eine Verschiebung derart vollzogen zu haben, dass sie ausschliesslich zu Armenversorgungsanstalten wurden. 1399 wurde zu Neustadt a. H. ein Beginenhaus ausdrücklich für arme Schwestern gestiftet, die um Brod gehen und am Rocken spinnen sollten, ${ }^{17}$ ) und der Lübecker Totentanz von 1496 lässt die Begine sprechen: Do mine vrunde my nicht konden ryke beraden, do makeden se van my eine baghinen. Durch die fortgesetzten Zuwendungen von Wohltātern bei festgelegter Mitgliederzahl gelangten trotzdem die Konvente nicht selten zu behaglichem Wohlstande, was bei dem Nachlassen der strengen Lebensweise zur Entartung führte, sodass die Reformation ohne besonderen Widerstand mit ihnen aufräumen konnte.

Zwar hatte man es pāpstlicherseits wie nach der ersten Verfolgung für nötig befunden, den Übereifer zu zügeln, und in zwei Bullen von 1374 und 1377 nahm Gregor XI. die "Armen beiderlei Geschlechts", welche durch sittliche und religiöse Führung keinen Anstoss erregten, in Schutz und befahl den Bischöfen, sie nicht wegen ihrer Kleidung belästigen zu lassen. ${ }^{19}$ ) Indessen scheint doch das Vorgehen des Erfurter Dominikaners in Sachsen und Thüringen dem Institut die Wurzeln abgegraben zu haben - die Nachrichten werden spārlicher, vor allem in Erfurt selbst, und nur die Hildesheimer Hâuser scheinen einigermassen die frühere Bedeutung gewahrt zu haben. Es bestanden dort bis jedenfalls Ende des fünfzehnten Jahrhunderts der alte, der neue Konvent und der im Johannesspital. Sie scheinen sich in günstiger Vermögenslage befunden zu haben, denn in allen dreien erwerben

16) Monum. Erphesfurt. ed. Holder-Egger.

17) Remling, Urkundenbuch der Bischöfe zu Speyer I nr. 679. 
Mitglieder Leibrenten, 1422 im ersten, 1448 im zweiten, 1404, 1419), 1464 im dritten. Auch mit der geistlichen Oberbehörde habem sie sich zu stellen gewusst, 1442 erteilt Bischof Magnus Ablas:s für die zur Ausbesserung ihres Hauses Beisteuernden..$^{18}$ ) In Halberstadt enwāhnt 1442 ein Vermāchtnis auch Schwestern, vermutlich Beginen, und 1465 verträgt sich das Paulsstift mit dera Rat und den Beginen über Wiesen in der faulen (jetzt Pfahl-) Gasse. In Magdeburg wird im fünfzehnten Jahrhundert noch eines Beginenhauses in der Jakobstrasse Erwähnung getan, vou dem sonst nichts bekannt ist, in Halle lag ein solches 1408 hinter dem Predigerkloster. ${ }^{19}$ )

Soweit die spärlichen Nachrichten Schlūsse zulassen, scheinen die Beginen ihre hergebrachten Beschäftigungen beibehalten zu haben; sie werden 1408 in Halle Klunkernonnen genannt, weil sie sich dürftig mit Spinnen nährten und eine 1485 von den dortigen Webern erhobene Beschwerde wegen Gewerbschmälerung nennt zwar die Schwestern von der dritten Regel des heiligen Franziskus, aber eben diese haben die Beginen vielfach angenommen. Ảhnliche Beobachtungen lassen sich anderswo machen. 1527 bestātigt Kardinal Albrecht die Stiftung eines Beginenhauses zu Aschaffenburg mit der Erlaubnis zwei Webstühle zu halten, dagegen wurde den beiden Beginenklausen zu Friedberg das Weben im sechszehnten Jahrhundert untersagt.80) Ihre Tätigkeit als Krankenpflegerinnen ist ausdrūcklich bezeugt im Hildesheimer Johannisspital; sie sollen über vierzig sein und gleiche, nicht grüne oder rote Kleidung tragen. In den erwähnten Aschaffenburger und Friedberger Ordnungen ist dafür eine bestimmte Taxe vorgesehen: 12 Pfennige, bezw. ein Turnos für Tag und Nacht. In Breslau waren dafür 3 Groschen und die Kost angesetzt. Naturgemäss schloss sich an diese Aufgabe die weitere, die Verstorbenen zu geleiten, mit der wir die Beginen schon früh betraut finden. Wohl hierauf bezieht sich die drastische Mitteilung aus Freiberg, wonach zwei Bürger in Strafe genommen

18) UB. von Hildesheim III nr. 1025 IV nr. 695, III nr. 127, 887; Staatsarchiv Hannover, Domstift Hildesheim 1808; UB. von Hildesheim IV nr, 449.

19) UB. von Halberstadt nr. 924, 1017, Magdeburger Geschichtsblātter II S. 26, 467.

20 St. A. Magdeburg Erzstift A II 156, May, Kardinal Albrecht I. Beilage 52a, Windhaus Kirche und Schule zu Friedberg (Archiv f. hess. Gesch. 1894.) 
werden, weil sie die Beginen "den huntslegern (Schindern) geglichet haben." ${ }^{21}$ ) Von Eulenspiegel berichtet das Volksbuch von 1515, dass er zu Mölln im Spital zum heiligen Geist von Beginen gepflegt und begraben worden sei, vor seinem Ende aber noch eine geärgert und befriedigt gesagt habe: "Es ist keine Begine so andächtig, wenn sie zornig wird, so ist sie ärger als der Teufel."

Die Neigung der Zeit zu derbem Spott hat den Gedankengang, der im Hildesheimer Johannesspital zu der Forderung vienzigjähriger Pflegerinnen führte, mit Vorliebe weiter ausgesponnen. Geiler von Kaisersberg meint: es ist ein misbrauch, Jas die jungen beginen zu den siechen gond, ja der siech tut inen nüt, es ist war, ist die frau siech, der man ist aber nit siech, ist der man siech, der knecht in dem hus ist nit siech oder der vetter, der zu siechen gat und kumpt lugen, wie er lebe. sie gond ouch etwan usz essen, es were besser, du schicktest inen heim. es solt den stab nieman an sich nemen under den frawen, sie wer denn vierzig jar alt, wiewol etlich sprechen sechzig jar. Noch deutlicher ist der grobe Murner in der Narrenbeschwörung:

noch blibt sie dannocht ein begin

und laszt sich schelten junkfrow drin.

und im Lutherisch Narr:

ich kann mich weder heben, legen und nit ein glid am leib me regen

ach bestel mir doch ein starke begein, doch dasz sie müsz ein junkfraw sein.

Dass die Ordnungen der Häuser immer eingehender werden, ist als günstiges Zeichen für die Entwicklung des innern Lebens nicht anzusehen. Gab doch 1393 Bischof Gerhard von Hildesheim dem alten Konvent eine solche gradezu zur Beseitigung vieler $Z$ wietracht. Danach war es schon nōtig, die Aufnahme berüchtigter Frauen, solcher mit kleinen Kindern, sowie Aussätziger zu verbieten. Wenn in der Bestätigung der Satzungen des neuen Konvents von 1401 durch Bischof Johann IIl. den Ausgestossenen untersagt wird, Geldforderungen zu erheben, so weist das auf ein nicht seltenes Vorkommnis hin. Auch sonst kehrt die Ermächti-

31) UB. von Hildesheim IV nr. 391, Schulz, Topographie Breslaus (Zeitschrift f. schles. Gesch. X), UB, von Freiberg III. 
gung zur Ausweisung unfriedlicher oder unsittlicher Elemente und das Verbot heimlichen Verkehrs ausser dem Hause regelmăssig wieder. Auch der Tracht schien es jetzt nötig Aufmerksamkeit zuzuwenden. Des Verbots für die Beginen des Hildesheimer Johannisspitals, rot und grün zu tragen, war schon oben Erwāhnung getan, die Lübecker von 1438 verbietet das Tragen von Mānteln mit Kragen, mit denen man viel Luxus zu treiben pflegte, und schrieb dafür Tũcher vor, ebensolche als Kopfbedeckung.") Was die Koketterie auch aus der Beginentracht machen konnte, darauf deutet Lauremberg in seinem zweiten Scherzgedicht:

De Börgerkinder even in sülker minen

Gehn nu als de verlopene Kloster-Beginen.

Auch die bei dem Zusammenleben grossenteils ungebildeter und müssiger Frauenspersonen unvermeidliche Ausartung des Unterhaltungstriebes hat frühzeitig Würdigung gefunden. Der Lübecker Totentanz hält der Begine vor:

Krichstu wat to weten gystern effte huede

Wo drade kumpt dat voert mank de luede.

„Beginentand und Altfrauenschnack" ist eine Redensart vom Ende des sechzehnten Jahrhunderts, und eine Weseler Ordnung ging sogar soweit, das Schweigen innerhalb des Hauses vorzuschreiben. ${ }^{23}$ ) Das bewog wohl auch Brant zu dem Wunsche:

Ach werent sy zu Portugall

Ach werents an derselben statt, Do der pfeffer gewachsen hat!

Je mehr in verānderten Zeiten das Beginenwesen überflüssig wurde, desto mehr schärfte sich der Blick für die Mängel der Einrichtung, und die Trägerin des einst ehrwürdigen, zeitweilig angefeindeten Namens wurde zur komischen Figur um so leichter, da die letzten Beginenhāuser als Altersversorgungsanstalten fortdauerten. So wird in Halberstadt noch 1794 ein Hospital zu den blauen Beginen erwähnt, gestiftet für zwölf alte Frauen, die blaue Kleidung trugen. Als der Kardinal Lang, Erzbischof von Salzburg

2) UB. v. Hildesheim II nr. 763, III nr. 13, UB. der Stadt Lübeck IX.

mann a. a. 0

2) Bruns, Beitrăge zur Bearbeitung alter Handschriften ff. 1802. S. 350; Heide- 
und vertrauter Ratgeber Maximilians I., auf der Augsburger Fastnacht sich als Begine verkleidet unter die Tanzenden mischte, war es ihm gewiss um den komischen Eindruck zu fun, und Pamphilus Gengenbach braucht den Ausdruck:

Und leit nit tag und nacht beim win,

So halt man in fur ein begin.

Noch 1652 meint der biedere kurbrandenburgische Ingenieur Schildknecht:

Es hat ein jede Begin im Spittel

Ihren eigen Husten und ihr Qual,

Bis sie der Tod würgt allzumal. ${ }^{24}$ )

21) Halberstädter Gemeinnūtzige Blātter 1794 I, S. 156, P. Qengenbach ed. Gödeke S. 58, Schildknecht Harmonia in forlalitiis, S. 237. 